19,18

\title{
Асимптотическое описание быстрых тепловых процессов в скалярных гармонических решетках
}

\author{
(С) Д.В. Кориков \\ Санкт-Петербургский политехнический университет Петра Великого, \\ Санкт-Петербург, Россия \\ E-mail: thecakeisalie@list.ru
}

Поступила в Редакцию 25 ноября 2019 г.

В окончательной редакции 20 марта 2020 г.

Принята к публикации 7 июля 2020 г.

Рассматриваются тепловые колебания в $d$-мерной $(d=1,2)$ скалярной гармонической решетке простой структуры. Описывается перераспределение осредненных кинетической и потенциальной энергий частиц после мгновенного теплового возбуждения („быстрый процесс“). Устанавливается, что при временах, много больших характерного периода атомных колебаний, разница кинетической и потенциальной энергий испытывает затухающие степенным образом осцилляции. Показатель степени в типичном случае равен $-d / 2$. Частоты осцилляций определяются из дисперсионного соотношения для решетки. Алгоритм доказательства допускает обобщение на скалярные и векторные решетки сложной структуры и различных размерностей. В качестве примера такого обобщения рассмотрены тепловые колебания в двумерной гексагональной решетке (решетка графена).

Ключевые слова: неравновесные тепловые процессы, скалярные решетки, гармонические кристаллы, кинетическая температура, графен.

DOI: $10.21883 /$ FTT.2020.11.50079.640

\section{1. Введение}

На микроуровне и в бездефектных слабонелинейных кристаллах (длина свободного пробега фононов много меньше характерного размера кристалла) процессы распространения тепла имеют не диффузионный (закон Фурье), а баллистический (волновой) характер. Описание неравновесных тепловых процессов в таких кристаллах является актуальной задачей физики твердого тела и востребовано во многих приложениях, связанных с нанотехнологиями. Удобной математической моделью для исследования тепловых процессов в твердых телах является гармонический кристалл (силы взаимодействия между частицами линейно зависят от их перемещений); такая модель часто используется в литературе [1-12,17-26]. Один из подходов к изучению таких кристаллов использует точные решения уравнений движения для описания статистических характеристик, таких как распределение вероятностей кинетической энергии или ее математическое ожидание („кинетическая температура“") [3,6,8-10,12,13,26]. Другой подход основан на исследовании динамики ковариаций и не требует знания точных решений. Для гармонических кристаллов замкнутая система уравнений для ковариаций перемещений и скоростей частиц может быть выведена из уравнений точной динамики частиц кристалла. В стационарной ситуации уравнения для ковариаций получены в $[12,14,15]$. Для описания нестационарных тепловых процессов в гармонических кристаллах вышеописанный подход развит в работах [17-20] (одномерный гармонический кристалл простой структуры) и [21-26] (двумерные скалярные решетки простой и сложной структуры).

В гармонических кристаллах при установлении термодинамического равновесия реализуется два вида тепловых процессов ${ }^{1}$. В ходе „быстрых“ процессов происходит локальное перераспределение энергии частиц между кинетической и потенциальной частями и между различными пространственными степенями свободы. Начальная разница между кинетической и потенциальной энергиями может быть вызвана, например, фемтосекундным ударным $([27-30])$ или лазерным ([31-35]) воздействием. При больших по сравнению с периодами колебаний атомов временах наступает локальное равновесие, при котором усредненная кинетическая энергия с большой точностью равна потенциальной, а распределения вероятностей для скоростей частиц становятся нормальными. Далее доминирующим становится „медленный“ процесс перераспределения кинетической температуры вдоль решетки.

Быстрые и медленные процессы в общей ситуации описаны в работах [24-26]; для медленных и быстрых процессов в континуальном приближении найдены интегральные выражения для кинетической температуры $T(t)$ и разницы $L(t)=K(t)-P(t)$ между средними кинетической $K(t)$ и потенциальной $P(t)$ энергиями. При однородном начальном распределении температур интегральное выражение для $L(t)$ является точным и может

\footnotetext{
${ }^{1}$ Разные моды колебаний гармонического кристалла не взаимодействуют друг с другом, поэтому мы не включаем в определение термодинамического равновесия равнораспределение механической энергии по модам колебаний.
} 
быть вычислено численно. Однако такая интегральная форма неудобна для анализа.

Настоящая работа посвящена описанию быстрых тепловых процессов при больших (по сравнению с периодами колебаний атомов) временах. Рассматривается бесконечная $d$-мерная $(d=1,2)$ гармоническая скалярная решетка простой структуры. Для такой решетки получены асимптотические выражения для разницы $L(t)$ кинетической и потенциальной энергий в форме

$$
L(t) \sim\left(\omega_{*} t\right)^{-\frac{d}{2}} T_{0}(\mathbf{r}) \sum_{s=1}^{m} c_{s} \cos \left(2 t \omega\left(\mathbf{k}_{s}\right)+\phi_{s}\right) .
$$

Здесь $\omega(\mathbf{k})$ есть дисперсионное соотношение для решетки (k - волновой вектор), а $\omega_{*}-$ характерная частота колебаний атомов. Формула (1) описывает осцилляции величины $L(t)$, амплитуда которых затухает, как $\left(\omega_{*} t\right)^{-d / 2}$. Частоты $2 \omega\left(\mathbf{k}_{s}\right)$ осцилляций определяются нулями $\mathbf{k}_{s}$ групповой скорости $\mathrm{d} \omega / \mathrm{d} \mathbf{k}$. Коэффициенты $c_{s}$ и $\phi_{s}$ описываются далее. Достаточными условиями для применимости формулы (1) являются

1. $\operatorname{det}\left(\mathrm{d}^{2} \omega / \mathrm{d}^{2} \mathbf{k}\right) \neq 0$ во всех точках $\mathbf{k}=\mathbf{k}_{s}$;

2. если $\omega(\mathbf{q})=0$, то $\left.\operatorname{det}\left[\mathrm{d}^{2} \omega^{2} / \mathrm{d}^{2} \mathbf{k}\right]\right|_{\mathbf{k}=\mathbf{q}} \neq 0$.

Поясним смысл этих условий. Условие 2 исключает из главного члена асимптотики $L(t)$ вклад от нулей функции $\omega(\mathbf{k})$. Это условие заведомо выполнено для любой решетки на упругом основании $\omega(\mathbf{k})$ не обращается в нуль) либо для любой решетки с парными силами притяжения (сила, действующая на частицу $\mathbf{x}$ со стороны частицы у имеет тот же знак, что и смещение частицы $\mathbf{y}$ относительно частицы х).

В статье приведены примеры одномерных и двумерных решеток с угловыми взаимодействиями, для которых амплитуда осцилляций затухает медленнее, чем $\left(\omega_{*} t\right)^{-d / 2}$. Кристаллы, для которых нарушено условие 1 , представляют интерес в связи с аномально медленным затуханием осцилляций кинетической и потенциальной энергий. Для одномерной цепочки необходимым условием аномального затухания является дальнодействие: чтобы амплитуда осцилляций затухала медленнее, чем $\left(\omega_{*} t\right)^{-1 / N}, N \gg 1$, необходимо, чтобы каждая частица кристалла взаимодействовала как минимум с $N$ coседями. При больших $N$ время затухания становится макроскопическим и после теплового возбуждения кристалл испытывает заметные наблюдателю пульсации температуры с заданной частотой.

Доказательство формулы (1) основано на известном многомерном методе стационарной фазы (см. [36]). Этот метод требует бесконечной дифференцируемости функции $\omega$ и гладкости границы области интегрирования. Благодаря периодичности функции $\omega$ вклады от углов области интегрирования взаимно сокращаются. Вклад от точек разрыва $\mathrm{d} \omega / \mathrm{d} \mathbf{k}$ вычисляется с помощью некоторых явных приемов, сводящих дело к одномерному методу стационарной фазы.

Для ряда одномерных (взаимодействия ближайших соседей, угловые взаимодействия, влияние упругого ос- нования) и двумерных (квадратная и треугольная решетки с парным взаимодействием, квадратная решетка с угловым взаимодействием) скалярных решеток простой структуры в работе явно выписаны асимптотические формулы. Кроме того, исследованы поперечные колебания гексагональной решетки, описано поведение кинетической температуры при больших временах. Примером материала с двумерной гексагональной решеткой является графен. Несмотря на то, что гексагональная решетка является решеткой со сложной структурой, для нее применимым тот же алгоритм, который был использован при выводе формулы (1). Это показывает, что полученные результаты могут быть обобщены на значительно более широкий класс решеток различной размерности.

\section{2. Скалярные решетки}

Рассмотрим $d$-мерную $(d=1,2)$ кристаллическую решетку. Элементарная ячейка содержит лишь одну частицу (,решетка простой структуры“). Предположим, что каждая частица имеет лишь одну степень свободы. Тогда положения частиц описываются скалярной функцией $u(\mathbf{x}, t)$, где $\mathbf{x}-$ радиус-вектор частицы в положении равновесия. Поэтому такая решетка называется скалярной.

Для гармонической решетки сила, действующая на частицу с равновесной координатой $\mathbf{x}$, представляет собой линейную комбинацию перемещений $u\left(\mathbf{y}_{\mathbf{z}}, t\right)$ всех частиц решетки. Здесь

$$
\mathbf{y}_{\mathbf{z}}=\mathbf{x}+\sum_{j=1}^{d} z_{j} \mathbf{e}_{j},
$$

где $\mathbf{e}_{j}, j=1, . ., d-$ базисные орты решетки, $z_{j}-$ целые числа и $\mathbf{z}=\left(z_{1}, \ldots, z_{d}\right)^{T}$. Уравнение движения частицы имеет вид

$$
\ddot{u}(\mathbf{x}, t)=\mathscr{L} u(\mathbf{x}, t), \quad \mathscr{L} u(\mathbf{x})=\omega_{*}^{2} \sum_{\mathbf{z}} b_{\mathbf{z}} u\left(\mathbf{y}_{\mathbf{z}}\right),
$$

где $\omega_{*}-$ характеристическая частота, коэффициент $b_{\mathbf{z}}$ определяет вклад частицы с координатой $\mathbf{y}_{\mathbf{z}}$ в суммарную силу, действующую на рассматриваемую частицу, $b_{-\mathbf{z}}=b_{\mathbf{z}}$. Будем рассматривать начальные условия

$$
u(\mathbf{x}, 0)=0, \quad \dot{u}(\mathbf{x}, 0)=v_{0}(\mathbf{x}),
$$

где $v_{0}(\mathbf{x})$ - независимые случайные величины с нулевыми математическим ожиданиями.

\section{3. Кинетическая температура}

При описании тепловых процессов в кристалле исследуется поведение статистических характеристик системы, таких как ковариации скоростей частиц. Сопоставим каждой частице с координатой $\mathbf{x}$ ее кинетическую температуру $T(\mathbf{x}, t):=k_{B}^{-1} m\left\langle\dot{u}(\mathbf{x}, t)^{2}\right\rangle$, где $m-$ масса частицы, 
$k_{B}$ - постоянная Больцмана. Через $\langle\ldots\rangle$ обозначается математическое ожидание. Условия (3) однозначно определяют кинетическую температуру в начальный момент времени $T(\mathbf{x}, 0)=k_{B}^{-1} m\left\langle v_{0}(\mathbf{x})^{2}\right\rangle$.

В работе [24] получены формулы, описывающие поведение кинетической температуры. Выпишем эти формулы для однородного начального распределения кинетической температуры $T(\mathbf{x}, 0)=T_{0}=$ const.

$$
\begin{gathered}
T(t)=\frac{T_{0}}{2}+\Delta T(t) \\
\Delta T(t)=\frac{T_{0}}{2(2 \pi)^{d}} \int_{[-\pi, \pi]^{d}} \cos (2 \omega(\mathbf{p}) t) \mathrm{d} \mathbf{p} .
\end{gathered}
$$

Здесь $\mathbf{p}=\left(p_{1}, \ldots, p_{d}\right)^{T}$ и

$$
\omega(\mathbf{p})^{2}=-\omega_{*}^{2} \sum_{\mathbf{z}} b_{\mathbf{z}} \cos (\mathbf{z} \cdot \mathbf{p})
$$

- дисперсионное соотношение для решетки. Формула (4) описывает колебания кинетической температуры, вызванные перераспределением между кинетической и потенциальной энергиями. Целью является описание поведения $T(t)$ при больших временах.

\section{4. Одномерная цепочка}

Пусть $d=1$. Поведение интеграла (4) исследуется методом стационарной фазы. Асимптотика $T(t)$ при $t \rightarrow+\infty$ определяется суммой вкладов от нулей $\mathrm{d} \omega / \mathrm{d} p$ („стационарных точек“), от концов отрезка интегрирования и от точек разрыва производных функции $\omega$ (,точки негладкости“). Поскольку подынтегральное выражение $2 \pi$ - периодическое, можно заменить промежуток интегрирования в (4) на $[q, q+2 \pi]$, где $\omega$ бесконечно дифференцируема в точке $q$ и $\mathrm{d} \omega(q) / \mathrm{d} p \neq 0$.

Покажем, что концы отрезка не дают вклад в асимптотику. Для этого представим функцию () в виде интеграла по бесконечному промежутку

$$
\Delta T(t)=\frac{T_{0}}{4 \pi} \int_{-\infty}^{+\infty} \cos (2 \omega(p) t) \chi(p-q) \mathrm{d} p .
$$

Здесь $\chi-$ некоторая бесконечно дифференцируемая функция, равная нулю везде, кроме конечного отрезка, причем $\chi=1$ в окрестности каждой из стационарных точек или точек негладкости. Пусть $\delta>0-$ достаточно мало и $\Psi$ - любая бесконечно дифференцируемая функция, такая что $\Psi(s)=1$ при $s<\delta / 2$ и $\Psi(s)=0$ при $s>\delta$. Тогда в качестве $\chi$ можно взять функцию, равную $1-\Psi(p)$ при $p<1 / 2$ и $\Psi(2 \pi-p)$ при $p \geq 1 / 2$. Для всякого $q$ и любой $2 \pi$-периодической функции $g(s)$ справедливо равенство

$$
\int_{q}^{q+2 \pi} g(s) \mathrm{d} s=\int_{-\infty}^{+\infty} \chi(s-q) g(s) \mathrm{d} s
$$

в частности, справедлива формула (6).
Теперь покажем, что выбором функции $\omega$ в (4) можно избавиться от вкладов точек негладкости. Функция $\omega$ бесконечно дифференцируема при $\omega(p)^{2} \neq 0$; в окрестности нуля $p_{0}$ функции $\omega^{2}$ справедливо разложение Тейлора

$$
\omega(p)^{2}=c_{k} \triangle p^{k}+c_{k+1} \triangle p^{k+1}+\ldots, \quad c_{k} \neq 0,
$$

где $\triangle p=p-p_{0}$. Поскольку $\omega$ вещественнозначная, старшая степень в разложении должна быть четной $k=2 l$. Тогда

$$
\omega(p)= \pm|\triangle p|^{l} \sqrt{c_{k}+c_{k+1} \triangle p+\ldots} .
$$

В (4) стоит четная функция от $\omega$. Поэтому выбором знака \pm в (8) можно так продолжить функцию $\omega$ при $p>p_{0}$, чтобы функция $\omega$ была гладкой в точке $p_{0}$. Повторяя ту же операцию для остальных нулей $\omega^{2}$, получаем бесконечно дифференцируемую функцию $\omega$.

Таким образом, в асимптотику $T(t)$ дают вклад лишь стационарные точки $p_{k} \in(q, q+2 \pi], k=1, \ldots, m$. Пусть $\alpha_{k}$ - такое, что $\omega^{(\alpha)}\left(p_{k}\right)=0$ при $\alpha<\alpha_{k}$ и $\omega^{\left(\alpha_{k}\right)}\left(p_{k}\right) \neq 0$.

Лемма 1 (лемма Эрдейи, см. лемму 3.1.2, [36]). Пусть $\alpha \geq 1$, бункция $f(x)$ бесконечно дифберенцируема при $x \geq 0$ и равна нулю при $x>a$. Тогда справедливо асимптотическое разложсение

$$
\begin{gathered}
\int_{0}^{+\infty} e^{i t x^{\alpha}} f(x) \mathrm{d} x \sim \sum_{k=0}^{\infty} a_{k} t^{-\frac{k+1}{\alpha}}, \quad t \rightarrow+\infty \\
a_{k}=\frac{f^{(k)}(0)}{k ! \alpha} \Gamma\left(\frac{k+1}{\alpha}\right) e^{\frac{i \pi(k+1)}{2 \alpha}} .
\end{gathered}
$$

Из последней леммы вытекает искомое разложение

$$
\Delta T(t) \sim \frac{T_{0}}{4 \pi} \operatorname{Re}\left(\sum_{l=1}^{m} e^{2 i \omega\left(p_{k}\right) t} \sum_{k=0}^{\infty} c_{l k} t^{-\frac{k+1}{\alpha_{k}}}\right), \quad t \rightarrow+\infty .
$$

Пример 1. Цепочка со взаимодействием ближайших соседей

$$
\mathscr{L} u(x)=\omega_{*}^{2}(u(x+a)-2 u(x)+u(x+a)),
$$

где а - равновесное расстояние межлуу соседними частицами, $\omega_{*}^{2}=\kappa / m(\kappa-$ жесткость связи). В этом случае кинетическая температура вычисляется явно (cM. [24]):

$$
\begin{aligned}
\Delta T(t) & =\frac{T_{0}}{2}\left(1+J_{0}\left(4 \omega_{*} t\right)\right) \\
& =\frac{T_{0}}{2}+\frac{T_{0}}{\sqrt{8 \pi \omega_{*} t}} \cos \left(4 \omega_{*} t-\pi / 4\right)+O\left(\frac{1}{\omega_{*} t}\right)
\end{aligned}
$$

при $t \rightarrow+\infty$.

Пример 2. Поперечные колебания цепочки со взаимодействием ближсайших соседей на упругом основании

$$
\mathscr{L} u(x)=\omega_{*}^{2}\left(u(x+a)-\left(2+\varepsilon^{2}\right) u(x)+u(x+a)\right),
$$


где $\omega_{*}^{2}=\kappa / m, \varepsilon^{2} \omega_{*}^{2}=\kappa_{f} / m u \kappa-$ жесткость связей между частицами, $\kappa_{f}-$ жесткость связи с основанием. Функция

$$
\omega(p)^{2}=2 \omega_{*}^{2}\left(1+\varepsilon^{2} / 2-\cos (p)\right)
$$

имеет на отрезке $[0,2 \pi)$ стационарные точки $p_{1}=0$, $p_{2}=\pi, \alpha_{1,2}=2$. Из леммы Эрдейи имеем

$$
\begin{aligned}
\Delta T(t) & =\frac{T_{0}}{4 \sqrt{\pi \omega_{*} t}}\left[\sqrt{\varepsilon} \cos \left(2 \omega_{*} \varepsilon t+\pi / 4\right)\right. \\
& \left.+\sqrt[4]{4+\varepsilon^{2}} \cos \left(2 \omega_{*} \sqrt{4+\varepsilon^{2}} t-\pi / 4\right)\right]+O\left(\frac{1+|\varepsilon|}{\omega_{*} t}\right)
\end{aligned}
$$

при $t \rightarrow+\infty$.

Пример 3. Поперечные колебания цепочки с угловыми взаимодействиями

$$
\begin{aligned}
\mathscr{L} u(x)= & -\omega_{*}^{2}(u(x+2 a)-4 u(x+a) \\
& +6 u(x)-4 u(x-a)+u(x-2 a)),
\end{aligned}
$$

где $\omega_{*}^{2}=\kappa_{a} / m$ и $\kappa_{a}-$ угловая жсесткость связи. В этом случае функция

$$
\omega(p)^{2}=2 \omega_{*}^{2}(3-4 \cos (p)+\cos (2 p))
$$

имеет на отрезке $0 \leq p<2 \pi$ стационарные точки $p_{1}=0, p_{2}=\pi, \alpha_{1,2}=2$. Тогда

$$
\Delta T(t)=\frac{T_{0}}{4 \sqrt{2 \pi \omega_{*} t}}\left(1+\sqrt{2} \cos \left(8 \omega_{*} t-\pi / 4\right)\right)+O\left(\frac{1}{\omega_{*} t}\right)
$$

при $t \rightarrow+\infty$.

Теперь приведем пример системы, в которой осцилляции кинетической температуры затухают медленнее, чем $1 / \sqrt{t}$.

Пример 4. Поперечные колебания цепочки с угловыми взаимодействиями на упругом основании

$$
\begin{aligned}
\mathscr{L} u(x)= & -\omega_{*}^{2}(u(x+2 a)-4 u(x+a) \\
& \left.+\left(6+\varepsilon^{2}\right) u(x)-4 u(x-a)+u(x-2 a)\right),
\end{aligned}
$$

где $\omega_{*}^{2}=\kappa_{a} / m, \varepsilon^{2} \omega_{*}^{2}=\kappa_{f} / m u \kappa_{a}-$ угловая жместкость связи между частицами, $\kappa_{f}-$ жесткость связи с основанием. Функция

$$
\omega(p)^{2}=2 \omega_{*}^{2}\left(3+\varepsilon^{2} / 2-4 \cos (p)+\cos (2 p)\right)
$$

имеет на отрезке $0 \leq p<2 \pi$ стационарные точки $p_{1}=0, \alpha_{1}=4$ и $p_{2}=\pi, \alpha_{2}=2$. Тогда

$$
\begin{aligned}
\Delta T(t)= & \frac{T_{0}}{8 \pi} \Gamma\left(\frac{1}{4}\right) \sqrt[4]{\frac{\varepsilon}{\omega_{*} t}} \cos \left(2 \varepsilon \omega_{*} t+\pi / 8\right) \\
& +O\left(\sqrt{\frac{1+|\varepsilon|}{\omega_{*} t}}\right)
\end{aligned}
$$

при $t \rightarrow+\infty$.

\section{5. Кристаллы с аномально медленным затуханием}

Формула (9) и пример 4 подсказывают нам, что могут существовать кристаллы, осцилляции кинетической и потенциальной энергий в которых ведут себя при $t \rightarrow+\infty$ как

$$
\Delta T(t) \sim T_{0} t^{-\delta} \cos \left(\omega_{\#} t+\varphi_{\#}\right), \quad 0<\delta \ll 1
$$

$\left(\omega_{\#}\right.$ и $\varphi_{\#}-$ некоторые коэффициенты). Такие системы мы будем называть кристаллами с аномально медленным затуханием. Поясним физический смысл условия (10). Оно означает, что среднее (по реализациям) отклонение кинетической энергии частицы в кристалле от равновесного значения испытывает очень медленно затухающие осцилляции. Усреднение по реализациям можно заменить усреднением по области кристалла, содержащей большое число частиц. Значит, температура кристалла после мгновенного теплового возбуждения осциллирует с частотой $\omega_{\#}$, очень медленно затухая.

Сформулируем некоторые условия, которым должны удовлетворять такие кристаллы. Для простоты ограничимся одномерным случаем. Тогда затухание осцилляций кинетической температуры описывается формулой (9). Условие (10) означает, что один из $\alpha_{k}$ в формуле (9) равен $1 / \delta$. Таким образом, на отрезке $[0,2 \pi)$ существует точка $p_{\#}$, в которой все производные $\omega$ вплоть до порядка $(1 / \delta)-1$ равны нулю. Для определенности будем считать, что $p_{\#}=0$. Тогда $\mathrm{d}^{l} \omega(p) / \mathrm{d} p^{l}=0$ для всех $l=1, \ldots,(1 / \delta)-1$. Пусть каждая частица взаимодействует с $2 M$ соседями, тогда коэффициенты $b_{k}$ в формуле (2) отличны от нуля только при $k=0, \pm 1, \ldots, \pm M$. Для простоты рассмотрим случай цепочки на упругом основании $-b_{0}>2 \sum_{k=1}^{M} b_{k}$; тогда функция $\omega^{2}(p)$ не имеет нулей и равенства $\mathrm{d}^{l} \omega(p) / \mathrm{d} p^{l}=0$ и $\mathrm{d}^{l} \omega^{2}(p) / \mathrm{d} p^{l}=0$ эквивалентны. Из выражения (5) очевидно, что все нечетные производные $\omega^{2}$ аннулируются в точке 0 . Для четных производных имеем

$$
\frac{\mathrm{d}^{2 l} \omega^{2}(0)}{\mathrm{d} p^{2 l}}=\mathrm{const} \sum_{k=1}^{M} b_{k} k^{2 l}
$$

Таким образом, условие $(10)$ для $\delta=1 / 2(L+1)$ выполняется тогда и только тогда, когда

$$
\sum_{k=1}^{M} b_{k} k^{2 l}=0, \quad l=1, \ldots, L .
$$

Эта система имеем нетривиальное решение если число уравнений $L$ меньше числа неизвестных $M$. Поэтому каждая частица в цепочке должна взаимодействовать не менее, чем с $2 M=2(L+1)=1 / \delta$ соседями. Таким образом, необходимым условием аномальности затухания является дальнодействие. Например, для того, чтобы амплитуда осцилляций кинетической температуры затухала как $t^{-1 / 100}$, требуется, чтобы каждая частица цепочки 
взаимодействовала как минимум со 100 ближайшими соседями.

Пример 5. Пусть $\delta=1 / 8$, тогда $L=3$ и минимальное число соседей, с которыми взаимодействует частица равно $2 M=2(L+1)=8$. Решением системы (11) является вектор

$$
\left(b_{1}, b_{2}, b_{3}, b_{4}\right)^{T}=\operatorname{const}(-56,28,-8,1)^{T} .
$$

Тогда $b_{0}=70+\varepsilon$. Уравнение движения (2) цепочки имеет вид

$$
\begin{aligned}
\ddot{u}(x) & =-\omega_{*}^{2}[u(x+4 a)-8 u(x+3 a)+28 u(x+2 a) \\
& -56 u(x+a)+\left(70+\varepsilon^{2}\right) u(x)-56 u(x+a) \\
& +28 u(x+2 a)-8 u(x+3 a)+u(x+4 a)] .
\end{aligned}
$$

Тогда $\omega_{\#}=\omega(0)=\omega_{*} \varepsilon$ и разложсение (9) при $t \rightarrow+\infty$ принимает вид

$\Delta T(t)=T_{0} \frac{\Gamma(1 / 8)}{16 \pi} \sqrt[8]{\frac{2 \varepsilon}{\omega_{*} t}} \cos \left(2 \varepsilon \omega_{*} t+\pi / 16\right)+O\left(\sqrt[4]{\frac{1}{\omega_{*} t}}\right)$.

Режим аномально медленного затухания неустойчив относительно малых изменений коэффициентов $b_{k}$ : при сколь угодно малом их изменении $b_{k} \rightarrow b_{k}+\delta b_{k}$ закон убывания амплитуды меняется с $\mathrm{C}(0) t^{-\delta}$ на $\mathrm{C}(\boldsymbol{\delta} \boldsymbol{b}) t^{-1 / 2}$ (через $\boldsymbol{\delta} \boldsymbol{b}$ обозначен вектор-столбец $\left.\left(\delta b_{1}, \ldots, \delta b_{M}\right)^{T}\right)$. Смена режима осуществляется следующим образом. Пусть $t^{a}(\boldsymbol{\delta} \boldsymbol{b})$ - момент наступления ,асимптотического“ режима, при котором $\Delta T(t)$ отличается от своей асимптотики не более, чем на $\varepsilon / 2,|\varepsilon| \ll 1$. Пусть $t_{m} \gg t^{a}(\mathbf{0})$. Поскольку точное решение системы (2) непрерывно зависит от $b_{k}$, можно выбрать такое достаточно малое $\tilde{\varepsilon}$, что при $|\boldsymbol{\delta} \boldsymbol{b}| \leq \tilde{\varepsilon}$ величины $\Delta T(t)$ для задач с коэффициентами $b_{k}$ и $b_{k}+\delta b_{k}$ отличаются не более, чем на $\varepsilon / 2,|\varepsilon| \ll 1$ на временном интервале $\left(0, t_{m}\right)$. Следовательно, при $t \in\left(t^{a}(0), t_{m}\right)$ амплитуда $\Delta T(t)$ отличается от $\mathrm{C}(0) t^{-\delta}$ не более чем на $\varepsilon$. В силу произвольности $\varepsilon$ и $t_{m}$ это означает, что $T_{A}$ и $\mathrm{C}_{\delta}$ стремятся к бесконечности при $\delta b_{k} \rightarrow 0$. Если $\delta b_{k}$ малы, время $t$ затухания осцилляций велико. Таким образом, медленное затухание разницы кинетической и потенциальной энергий сохраняется и при малых отклонениях коэффициентов $b_{k}$ от решений системы (11).

\section{6. Двумерная решетка}

В случае $d=2$ для описания поведения $T(t)$ при больших временах используется многомерный метод стационарной фазы. Аналогично одномерному случаю, вклад в асимптотику $T(t)$ при $t \rightarrow+\infty$ определяется суммой вкладов от нулей $\nabla \omega$, точек разрыва производных функции $\omega$ и границы области интегрирования.

Функция (5) периодическая по $p_{1,2}$, поэтому область интегрирования в формуле (4) можно заменить на любой квадрат $\Pi:=\left\{0 \leq p_{j}+\theta_{j} \leq 2 \pi\right\}$. Выберем $\theta_{j}$ так, чтобы ни один из нулей $\omega$ или $\nabla \omega$ не лежал на границе квадрата П. Функция (5) гладкая в П, за исключением конечного числа точек $\mathbf{q}^{1}, \ldots, \mathbf{q}^{l}$ (нулей $\left.\omega^{2}\right)$. Квадрат П содержит конечное число нулей $\mathbf{p}^{1}, \ldots, \mathbf{p}^{m}$ вектора групповой скорости $\nabla \omega$. Будем считать, что $\operatorname{det} \nabla^{2} \omega\left(\mathbf{p}^{k}\right) \neq 0$, $k=1, \ldots, m$.

Сначала покажем, что граница области интегрирования не дает вклада в асимптотику $T(t)$. Для этого воспользуемся формулой (7) и перепишем правую часть (4) в виде интеграла по всей плоскости

$$
\frac{T_{0}}{4 \pi} \operatorname{Re} \int_{-\infty}^{\infty} \int_{-\infty}^{\infty} e^{2 i \omega(\mathbf{p}) t} \chi\left(p_{1}-\theta_{1}\right) \chi\left(p_{2}-\theta_{2}\right) \mathrm{d} p_{1} \mathrm{~d} p_{2} .
$$

Отметим, что $\chi\left(p_{1}-\theta_{1}\right) \chi\left(p_{2}-\theta_{2}\right)=1$ вблизи точек $\mathbf{p}^{k}, \mathbf{q}^{k}$.

Рассмотрим вклады от стационарных точек $\mathbf{p}^{k}$ и от точек негладкости $\mathbf{q}^{k}$ по отдельности. Для этого перепишем выражение (13) в виде суммы

$$
\Delta T(t)=\frac{T_{0}}{4 \pi} \operatorname{Re}\left[I(t)+\sum_{k=1}^{l} \tilde{I}_{k}(t)\right] .
$$

Здесь

$$
\tilde{I}_{k}(t):=\int_{-\infty}^{\infty} \int_{-\infty}^{\infty} e^{2 i \omega(\mathbf{p}) t} \Psi\left(\left|\mathbf{p}-\mathbf{q}^{k}\right|\right) \mathrm{d} p_{1} \mathrm{~d} p_{2},
$$

и функция $\Psi$ определена после формулы (). Интегрирование в (14) ведется фактически по малому кругу с центром в точке $\mathbf{q}^{k}$. Поэтому $\tilde{I}_{k}(t)$ определяет вклады от точки негладкости $\mathbf{q}^{k}$. Интеграл

$$
\begin{gathered}
I(t):=\int_{-\infty}^{\infty} \int_{-\infty}^{\infty} e^{2 i t \omega(\mathbf{p})} f(\mathbf{p}) \mathrm{d} p_{1} \mathrm{~d} p_{2} \\
f(\mathbf{p}):=\chi\left(p_{1}-\theta_{1}\right) \chi\left(p_{2}-\theta_{2}\right)-\sum_{k=1}^{l} \Psi\left(\left|\mathbf{p}-\mathbf{q}^{k}\right|\right)
\end{gathered}
$$

определяет вклад от всех стационарных точек $\mathbf{p}^{k}$. Функция $f$ обращается в нуль вблизи точек $\mathbf{q}^{k}$, поэтому интегрирование ведется фактически по области, в которой групповая скорость $\omega$ бесконечно дифференцируема. Благодаря этому можно воспользоваться обычным многомерным методом стационарной фазы (см. лемму 3.2.1, теорему 3.2.1, [36]) и получить для $I(t)$ разложение

$$
I(t)=\sum_{k=1}^{m} \frac{c_{k} e^{i\left(2 t \omega\left(\mathbf{p}^{k}\right)+\phi_{k}\right)}}{t}+O\left(t^{-2}\right), \quad t \rightarrow+\infty .
$$

Коэффициенты $c_{k}$ однозначно определяются по $\omega$; в частности,

$$
c_{k}=\pi\left|\operatorname{det} \nabla^{2} \omega\left(\mathbf{p}^{k}\right)\right|^{-1 / 2}, \quad \phi_{k}=\pi \operatorname{sgn} \nabla^{2} \omega\left(\mathbf{p}^{k}\right) / 4 .
$$

(через $\operatorname{sgn} A$ обозначается разница между числом положительных и числом отрицательных собственных значений матрицы $A)$. 
Выясним достаточные условия, при которых можно пренебречь вкладом от точек негладкости $\mathbf{q}^{k}$. Разложим $\omega^{2}(\mathbf{p})$ в ряд Тейлора

$$
\omega^{2}(\mathbf{p})=(\mathbf{a} \cdot \Delta \mathbf{p})+(A \Delta \mathbf{p} \cdot \Delta \mathbf{p})+\ldots
$$

вблизи точки $\mathbf{q}^{k}$; здесь $\Delta \mathbf{p}:=\mathbf{p}-\mathbf{q}^{k}$. Поскольку функция $\omega(\mathbf{p})$ вещественна, $\mathbf{a}=0$ и матрица $A$ неотрицательна. Будем считать, что матрица $A$ положительно определена. Это предположение выполняется для любой решетки с парным взаимодействием

$$
\begin{gathered}
\mathscr{L} u(\mathbf{x})=\omega_{*}^{2} \sum_{\mathbf{z} \neq \mathbf{0}} a_{\mathbf{z}}\left(u\left(\mathbf{y}_{\mathbf{z}}\right)-u\left(\mathbf{y}_{\mathbf{0}}\right)\right), \quad a_{\mathbf{z}}=a_{-\mathbf{z}} \geq 0 ; \\
\omega(\mathbf{p})^{2}=\omega_{*}^{2} \sum_{\mathbf{z} \neq \mathbf{0}} a_{\mathbf{z}}(1-\cos (\mathbf{z} \cdot \mathbf{p})) .
\end{gathered}
$$

В самом деле, тогда нулями $\omega^{2}(\mathbf{p})$ являются точки $\mathbf{q}:=\left(2 \pi k_{1}, 2 \pi k_{2}\right)$ и () принимает вид

$$
\omega^{2}(\mathbf{p})=\omega_{*}^{2} \sum_{\mathbf{z} \neq \mathbf{0}} a_{\mathbf{z}}(\mathbf{z} \cdot \Delta \mathbf{p})^{2} / 2+\ldots
$$

Среди векторов $\mathbf{z}, a_{\mathbf{z}}$ есть хотя бы пара неколлиенарных (в противном случае решетка распадается на независимые одномерные цепочки). Поэтому квадратичное слагаемое в (18) положительно при $\Delta \mathbf{p} \neq \mathbf{0}$ и матрица $A$ положительно определена.

По лемме Морса (см. лемму 2.3.1, [36]), при сделанных предположениях существует такая бесконечно дифференцируемая замена переменных $\mathbf{p} \rightarrow z_{1}(\mathbf{p}), z_{2}(\mathbf{p})$, что $\omega^{2}(\mathbf{p})=z_{1}^{2}+z_{2}^{2}=r^{2}$ вблизи $\mathbf{q}^{k}$. Тогда переходом к полярным координатам $z_{1}=r \cos (\varphi), z_{2}=r \sin (\varphi)$ в (14) получаем

$$
\tilde{I}_{k}(t):=\int_{0}^{\infty} e^{2 i r t} r\left(\int_{0}^{2 \pi} \Phi(r, \varphi) \mathrm{d} \varphi\right) \mathrm{d} r .
$$

Функция $\Phi(r, \varphi)=\Psi(|\Delta \mathbf{p}|) J\left(z_{1}, z_{2}\right)$ (где $J-$ якобиан замены $\left.\mathbf{p} \rightarrow z_{1}, z_{2}\right)$ является бесконечно дифференцируемой при $r \geq 0, \varphi \in[0,2 \pi]$. Рассмотрим выражение (19), как однократный интеграл по $r$ и применим лемму Эрдейи; получим $\tilde{I}_{k}(t)=O\left(t^{-2}\right)$. Таким образом, вклад от $\tilde{I}_{k}(t)$ пренебрежимо мал по сравнению с главным членом (16), и справедливо разложение

$$
\Delta T(t)=\frac{T_{0}(\mathbf{r})}{4 \pi t} \sum_{k=1}^{m} c_{k} \cos \left(2 t \omega\left(\mathbf{p}^{k}\right)+\phi_{k}\right)+O\left(\frac{1}{\omega_{*}^{2} t^{2}}\right)
$$

при $\omega_{*} t \rightarrow \infty$.

Пример 6. Поперечные колебания квадратной решетки со взаимодействием ближсайших соседей

$$
\begin{aligned}
\mathscr{L} u(\mathbf{x})= & \omega_{*}^{2}\left(u\left(\mathbf{x}+\mathbf{e}_{1}\right)+u\left(\mathbf{x}+\mathbf{e}_{2}\right)-4 u(\mathbf{x})\right. \\
& \left.+u\left(\mathbf{x}-\mathbf{e}_{1}\right)+u\left(\mathbf{x}-\mathbf{e}_{2}\right)\right),
\end{aligned}
$$

где $\mathbf{e}_{1}=(1,0)^{T} u \mathbf{e}_{2}=(0,1)^{T}$. Тогда

$$
\omega^{2}(\mathbf{p})=2 \omega_{*}^{2}\left(2-\cos \left(p_{1}\right)-\cos \left(p_{2}\right)\right) .
$$

В квадрате $\Pi:=\left\{0 \leq p_{j}+\pi / 2 \leq 2 \pi\right\}$ расположены нули $\mathbf{p}^{1}=(0, \pi), \mathbf{p}^{2}=(\pi, 0), \mathbf{p}^{3}=(\pi, \pi)$ функиии $\nabla \omega$ и нуль $\mathbf{q}^{1}=(0,0)$ функиии $\omega^{2}$, причем $\omega^{2}=$ $=\omega_{*}^{2}\left(p_{1}^{2}+p_{2}^{2}\right)+\ldots$ при $\mathbf{p} \rightarrow(0,0)$. Разложение имеет вид

$$
\begin{aligned}
& \Delta T(t)=\frac{T_{0}}{2 \omega_{*} t} \\
& \times\left(\frac{1}{\sqrt{2}} \sin \left(4 \sqrt{2} \omega_{*} t\right)+\cos \left(4 \omega_{*} t\right)\right)+O\left(\frac{1}{\omega_{*}^{2} t^{2}}\right) .
\end{aligned}
$$

Пример 7. Поперечные колебания треугольной решетки со взаимодействием ближайших соседей

$$
\begin{aligned}
& \mathscr{L} u(\mathbf{x})=\omega_{*}^{2}\left(u\left(\mathbf{x}+\mathbf{e}_{1}\right)+u\left(\mathbf{x}+\mathbf{e}_{2}\right)+u\left(\mathbf{x}+\mathbf{e}_{1}-\mathbf{e}_{2}\right)\right. \\
&\left.\left.-6 u(\mathbf{x})+u\left(\mathbf{x}-\mathbf{e}_{1}\right)+u\left(\mathbf{x}-\mathbf{e}_{2}\right)\right)+u\left(\mathbf{x}-\mathbf{e}_{1}+\mathbf{e}_{2}\right)\right), \\
& \text { где } \mathbf{e}_{1}=(1,0)^{T} u \mathbf{e}_{2}=(1 / 2, \sqrt{3} / 2)^{T} . \text { Тогда } \\
& \omega^{2}(\mathbf{p})=2 \omega_{*}^{2}\left(3-\cos \left(p_{1}\right)-\cos \left(p_{2}\right)-\cos \left(p_{1}-p_{2}\right)\right) .
\end{aligned}
$$

В квадрате $\Pi:=\left\{0 \leq p_{j}+\pi / 2 \leq 2 \pi\right\}$ расположены нули $\mathbf{p}^{1}=(0, \pi), \mathbf{p}^{2}=(\pi, 0), \mathbf{p}^{3}=(\pi, \pi), \mathbf{p}^{4}=(2 \pi / 3,4 \pi / 3)$, $\mathbf{p}^{4}=(4 \pi / 3,2 \pi / 3)$ функции $\nabla \omega$ и нуль $\mathbf{q}^{1}=(0,0)$ функиии $\omega^{2}$, причем

$$
\omega^{2}=\omega_{*}^{2}\left(\left(p_{1}-p_{2} / 2\right)^{2}+\left(p_{2} \sqrt{3} / 2\right)^{2}\right)+\ldots, \quad \mathbf{p} \rightarrow(0,0) .
$$

Разложение (20) имеет вид

$$
\begin{aligned}
& \Delta T(t)=\frac{T_{0}}{\omega_{*} t} \\
& \times\left(\frac{3}{\sqrt{2}} \cos \left(4 \sqrt{2} \omega_{*} t\right)+2 \sqrt{3} \sin \left(6 \omega_{*} t\right)\right)+O\left(\frac{1}{\omega_{*}^{2} t^{2}}\right) .
\end{aligned}
$$

Следующий пример показывает, что при нарушении условия невырожденности $\operatorname{det} \nabla^{2} \omega\left(\mathbf{p}^{k}\right) \neq 0$, $k=1, \ldots, M$ формула (20), вообще говоря, неверна, а амплитуда осцилляций $\Delta T$ может затухать медленнее, чем $O(1 / t)$.

Пример 8. Поперечные колебания квадратной решетки с угловыми взаимодействиями вида

$$
\begin{aligned}
& \mathscr{L} u(\mathbf{x})=-\omega_{*}^{2}\left(u\left(\mathbf{x}+2 \mathbf{e}_{1}\right)-4 u\left(\mathbf{x}+\mathbf{e}_{1}\right)\right. \\
& \quad+6 u(\mathbf{x})-4 u\left(\mathbf{x}-\mathbf{e}_{1}\right)+u\left(\mathbf{x}-2 \mathbf{e}_{1}\right)+u\left(\mathbf{x}+2 \mathbf{e}_{2}\right) \\
& \left.\quad-4 u\left(\mathbf{x}+\mathbf{e}_{2}\right)+6 u(\mathbf{x})-4 u\left(\mathbf{x}-\mathbf{e}_{2}\right)+u\left(\mathbf{x}-2 \mathbf{e}_{2}\right)\right),
\end{aligned}
$$

(здесь угловые пружсины соединяют только такие тройки соседних частии, которые лежсат на одной вертикальной или горизонтальной прямой). Тогда

$$
\begin{aligned}
\omega^{2}(\mathbf{p})= & 2 \omega_{*}^{2}\left(6-4 \cos \left(p_{1}\right)-4 \cos \left(p_{2}\right)\right. \\
& \left.+\cos \left(2 p_{1}\right)+\cos \left(2 p_{2}\right)\right) .
\end{aligned}
$$


В квадрате $\Pi:=\left\{0 \leq p_{j}+\pi / 4 \leq 2 \pi\right\}$ расположсены нули $(0,0), \quad(0, \pi), \quad(\pi, 0), \quad(\pi, \pi)$ бункции $\nabla \omega u$ нуль $\mathbf{q}_{1}=(0,0)$ бункции $\omega^{2}$, причем $\operatorname{det} \nabla^{2} \omega(0, \pi)=$ $=\operatorname{det} \nabla^{2} \omega(\pi, 0)=0 u \operatorname{det} \nabla^{2} \omega(\pi, \pi) \neq 0$. Опишем вклад в асимптотику $\Delta T(t)$ от вырожденных стационарных точек $\mathbf{p}_{1}=(\pi, 0), \mathbf{p}_{2}=(0, \pi)$. В силу Леммы 3.5.1, [36] существует такая гладкая замена переменных $\mathbf{p} \rightarrow z_{1, k}(\mathbf{p}), z_{2, k}(\mathbf{p})$, что $z_{1, k}\left(\mathbf{p}_{k}\right)=z_{2, k}\left(\mathbf{p}_{k}\right)=0$, $\omega(\mathbf{p})=\omega_{*}\left(4-z_{1, k}^{2}+z_{2, k}^{4} / 8\right)$ в окрестности точки $\mathbf{p}_{k}$ и якобиан замены $J\left(z_{1, k}, z_{2, k}\right)$ обращается в единииу в точке $\left(z_{1, k}, z_{2, k}\right)=(0,0)$. Тогда

$$
\begin{aligned}
& I_{k}(t):=\int_{-\infty}^{+\infty} \int_{-\infty}^{+\infty} e^{2 i \omega(\mathbf{p}) t} \Psi\left(\left|\mathbf{p}-\mathbf{p}_{k}\right|\right) d p_{1} d p_{2} \\
& =e^{8 i \omega_{*} t} \int_{-\infty}^{+\infty} d z_{2, k} e^{i z_{2, k}^{4} \omega_{*} t / 4} \int_{-\infty}^{+\infty} e^{-2 i z_{1, k}^{2} \omega_{*} t} f\left(z_{1, k}, z_{2, k}\right) d z_{1, k} .
\end{aligned}
$$

Функция $z_{1, k}, z_{2, k} \rightarrow f_{k}\left(z_{1, k}, z_{2, k}\right):=\Psi\left(\left|\mathbf{p}\left(z_{1, k}, z_{2, k}\right)-\mathbf{p}_{k}\right|\right)$ $\times J\left(z_{1, k}, z_{2, k}\right)$ является гладкой, причем $f_{k}(0,0)=1$. Применяя метод стационарной базы (Теорема 3.1.6, [36]), выводим равномерную по $z_{2, k}$ асимптотику

$$
\begin{aligned}
& \int_{-\infty}^{+\infty} e^{-2 i z_{1, k}^{2} \omega_{*} t} f_{k}\left(z_{1, k}, z_{2, k}\right) d z_{1, k} \\
& =f_{k}\left(0, z_{2, k}\right) e^{i \pi / 4} \sqrt{\frac{\pi}{2 \omega_{*} t}}+O\left(\left(\omega_{*} t\right)^{-3 / 2}\right) .
\end{aligned}
$$

В силу леммы Эрдейи,

$$
\begin{aligned}
& \int_{-\infty}^{+\infty} e^{i z_{2, k}^{4} \omega_{*} t / 4} f_{k}\left(0, z_{2, k}\right) d z_{2, k} \\
& =\frac{e^{i \pi / 8}}{2 \sqrt{2}} \Gamma\left(\frac{1}{4}\right)\left(\omega_{*} t\right)^{-1 / 4}+O\left(\frac{1}{\sqrt{\omega_{*} t}}\right) .
\end{aligned}
$$

Комбинируя две последние формуль, получаем

$$
\begin{aligned}
I_{1}(t)+I_{2}(t)= & \frac{\sqrt{\pi}}{2} \Gamma\left(\frac{1}{4}\right)\left(\omega_{*} t\right)^{-3 / 4} e^{8 i \omega_{*} t+3 i \pi / 8} \\
& +O\left(\left(\omega_{*} t\right)^{-1 / 2}\right) .
\end{aligned}
$$

Оценим вклад в асимптотику $\Delta T(t)$ от точки негладкости $(0,0)$. Ряд Тейлора (17) для $\omega^{2}$ в окрестности точки $(0,0)$ имеет вид $\omega^{2}(\mathbf{p})=\omega_{*}^{2}\left(p_{1}^{4}+p_{2}^{4}\right)+\ldots$ По лемме Морса, существует такая гладкая замена переменных $\mathbf{p} \rightarrow z_{1}(\mathbf{p}), z_{2}(\mathbf{p})$, что $\omega^{2}(\mathbf{p})=z_{1}^{4}+z_{2}^{4}$ вблизи точки $\mathbf{p}=(0,0)$. Пусть

$$
\tilde{I}_{1}(t)=\int_{-\infty}^{\infty} \int_{-\infty}^{\infty} e^{2 i \omega_{*} \sqrt{z_{1}^{4}+z_{2}^{4}} t} \Psi(|\mathbf{p}|) J\left(z_{1}, z_{2}\right) \mathrm{d} z_{1} \mathrm{~d} z_{2}
$$

После замены $z_{1}= \pm \sqrt{r|\cos \varphi|}, z_{2}= \pm \sqrt{r|\sin \varphi|}$ интеграл $\tilde{I}(t)$ принимает вид

$$
\tilde{I}_{1}(t)=\int_{0}^{\infty} e^{2 i \omega_{*} r t} F(r) \mathrm{d} r
$$

Здесь

$$
F(r):=8 \int_{0}^{2 \pi} \frac{\Psi(|\mathbf{p}|) J\left(z_{1}, z_{2}\right)}{\sqrt{|2 \sin (2 \varphi)|}} \mathrm{d} \varphi, \quad F(0)=32 K\left(\frac{1}{2}\right),
$$

где $K$ - полный эллиптический интеграл Лежсандра первого рода, $K(1 / 2) \simeq 1.854$. Из леммы Эрдейи следует, что

$$
\tilde{I}_{1}(t)=\frac{i F(0)}{2 \omega_{*} t}+O\left(\frac{1}{\omega_{*}^{2} t^{2}}\right)
$$

Стационарная точка $(\pi, \pi)$ невырождена $\operatorname{det} \nabla^{2} \omega(\pi, \pi) \neq 0$, поэтому

$$
\begin{gathered}
\int_{-\pi}^{\pi} \int_{-\pi}^{\pi} e^{2 i \omega(\mathbf{p}) t}\left(1-\sum_{k=1,2} \Psi\left(\left|\mathbf{p}-\mathbf{p}_{k}\right|\right)-\Psi(|\mathbf{p}|)\right) d p_{1} d p_{2} \\
=O\left(\left(\omega_{*} t\right)^{-1}\right)
\end{gathered}
$$

(ср. с формулой (16)). Комбинируя формулы (23), (24) u (25), получаем

$$
\begin{aligned}
\Delta T(t)= & \frac{T_{0} \Gamma(1 / 4) \sqrt{\pi}}{16 \pi^{2}} \cos \left(8 \omega_{*} t+3 \pi / 8\right)\left(\omega_{*} t\right)^{-3 / 4} \\
& +O\left(\frac{1}{\sqrt{\omega_{*} t}}\right), \quad t \rightarrow+\infty
\end{aligned}
$$

При моделировании реальных кристаллических решеток взаимодействия между частицами, как правило, не бывают чисто угловыми. Таким образом, в типичных ситуациях применима формула (20).

\section{7. Гексагональная решетка}

Гексагональная решетка является решеткой сложной структуры (элементарная ячейка содержит 2 частицы). Примером материала с такой решеткой является графен. В работе [25] найдено выражение для колебаний кинетической температуры

$$
T=\frac{T_{0}}{2}\left[1+\frac{1}{8 \pi^{2}} \int_{0}^{2 \pi} \int_{0}^{2 \pi}\left[\cos \left(2 \omega_{+} t\right)+\cos \left(2 \omega_{-} t\right)\right] \mathrm{d} p_{1} \mathrm{~d} p_{2}\right] .
$$

Здесь

$$
\omega_{ \pm}=\omega_{*} \sqrt{3 \pm \sqrt{3+2\left(\cos p_{1}+\cos p_{2}+\cos \left(p_{1}-p_{2}\right)\right)}} .
$$


Показатели затухания и характерные частоты для различных гармонических решеток

\begin{tabular}{c|c|c|c}
\hline № & Тип решетки & $d$ & $\begin{array}{c}\text { Частоты осцилляций } \\
\text { кинетической температуры, } s^{-1}\end{array}$ \\
\hline
\end{tabular}

\begin{tabular}{l|l|r|r}
\hline \multicolumn{2}{c}{ Одномерные решетки } & \multicolumn{2}{c}{ - } \\
\hline 1 & Цепочка с ВБС & $1 / 2$ & $2 \omega_{*} \varepsilon, 2 \omega_{*} \sqrt{4+\varepsilon^{2}}$ \\
2 & Цепочка с ВБС на УО & $1 / 2$ & $0,8 \omega_{*}$ \\
3 & Цепочка с УВ & $1 / 2$ & $2 \varepsilon \omega_{*}$ \\
4 & Цепочка с УВ на УО & $1 / 4$ & $2 \varepsilon \omega_{*}$ \\
5 & Цепочка с законом взаимодействия (12) & $1 / 8$ & \\
\hline
\end{tabular}

\begin{tabular}{l|l}
\hline \multicolumn{2}{|l}{} \\
\hline 6 & Квадратная решетка с ВБС \\
7 & Треугольная решетка с ВБС \\
8 & Квадратная решетка с УВ \\
9 & Решетка графена
\end{tabular}

Двумерные решетки

Принятые сокращения и обозначения: № - номер примера; $d-$ показатель степенного затухания $\left(\omega_{*} t\right)^{-d}$ амплитуды колебаний кинетической температуры. ВБС - взаимодействия ближайших соседей; УВ - угловые взаимодействия; УО - упругое основание; $\omega_{*}$ и $\omega_{*} \varepsilon-$ характерные частоты атомных колебаний $\omega_{*}^{2}=k_{p, a} / m,\left(\omega_{*} \varepsilon\right)^{2}=k_{f} / m$, где $k_{p}$ - жесткость связи между соседними частицами, $k_{a}-$ жесткость угловой связи, $k_{f}$ - жесткость связи с основанием, $m$ - масса частицы.

Опишем поведение кинетической температуры (26) при больших временах. Обозначим

$$
J_{ \pm}(t):=\int_{0}^{2 \pi} \int_{0}^{2 \pi} e^{2 i \omega_{ \pm} t} \mathrm{~d} p_{1} \mathrm{~d} p_{2}
$$

тогда

$$
J_{ \pm}(t)=\int_{-\infty}^{+\infty} \int_{-\infty}^{+\infty} e^{2 i \omega_{ \pm} t} \chi\left(p_{1}+\pi / 3\right) \chi\left(p_{2}+\pi / 3\right) \mathrm{d} p_{1} \mathrm{~d} p_{2}
$$

(функция $\chi$ - такая же, как в формуле (7)). Вклад в асимптотику $J_{ \pm}(t)$ при $t \rightarrow+\infty$ дают стационарные точки и точки негладкости функции $\omega_{ \pm}$, принадлежащие фактической области интегрирования $\chi\left(p_{1}+\pi / 3\right) \chi\left(p_{2}+\pi / 3\right) \neq 0$. Перечислим все такие точки. Седловые точки функций $\omega_{ \pm}$есть $(0, \pi),(\pi, 0)$ и $(\pi, \pi)$. Точки $(2 \pi / 3,4 \pi / 3)$ и $(4 \pi / 3,2 \pi / 3)$ являются точками негладкости функций $\omega_{ \pm}$. Точка $(0,0)$ является стационарной точкой (точкой максимума) для $\omega_{+}$и точкой негладкости функции $\omega_{-}$.

Покажем, что вклады от точек негладкости убывают, как $O\left(t^{-2}\right)$. Обозначим

$$
A(\mathbf{p}):=3+2\left(\cos p_{1}+\cos p_{2}+\cos \left(p_{1}-p_{2}\right)\right) .
$$

Вблизи точки $\mathbf{P}:=(2 \pi / 3,4 \pi / 3)$ справедливо разложение Тейлора

$$
\begin{gathered}
A(\mathbf{p})=\left(\Delta p_{1}-\Delta p_{2} / 2\right)^{2}+3 \Delta p_{2}^{2} / 4+\ldots, \\
\Delta p_{1}=p_{1}-2 \pi / 3, \quad \Delta p_{2}=p_{2}-4 \pi / 3 .
\end{gathered}
$$

По лемме Морса, существует такая гладкая замена переменных $p_{1}, p_{2} \rightarrow q_{1}, q_{2}$ с якобианом $\mathfrak{J}$, что $A(\mathbf{p})=q_{1}^{2}+q_{2}^{2}$ в окрестности точки $(2 \pi / 3,4 \pi / 3)$. Поэтому

$$
\begin{aligned}
& \int_{-\infty}^{+\infty} \int_{-\infty}^{+\infty} e^{2 i \omega_{ \pm} t} \chi(|\mathbf{p}-\mathbf{P}|) \mathrm{d} p_{1} \mathrm{~d} p_{2} \\
& =\int_{0}^{+\infty} \int_{0}^{2 \pi} e^{2 i \omega_{*} t \sqrt{3 \pm r}} \mathfrak{J}\left(q_{1}, q_{2}\right) \chi(|\mathbf{p}-\mathbf{P}|) r \mathrm{~d} \varphi \mathrm{d} r \\
& =\int_{0}^{+\infty} e^{2 i \omega_{*} t \sqrt{3 \pm r}} \mathfrak{F}(r) r \mathrm{~d} r
\end{aligned}
$$

Здесь $(r, \varphi)$ - полярные координаты с началом в точке $\left(q_{1}, q_{2}\right)=(0,0)$ и функция

$$
\mathfrak{F}(r)=\int_{0}^{2 \pi} \mathfrak{J}\left(q_{1}, q_{2}\right) \chi(|\mathbf{p}-\mathbf{P}|) \mathrm{d} \varphi
$$

является гладкой при $r \geq 0$ и аннулируется вдали от нуля. После замены переменных $r= \pm x(x+2 \sqrt{3})$ интеграл (28) принимает вид

$$
2 e^{2 i \omega_{*} \sqrt{3} t} \int_{0}^{+\infty} e^{2 i \omega_{*} x t} \mathfrak{F}(r) x(x+\sqrt{3}) x(x+2 \sqrt{3}) \mathrm{d} x
$$

и по лемме Эрдейи убывает как $O\left(t^{-2}\right)$. Меняя ролями $p_{1}$ и $p_{2}$, получаем такой же результат для точки $(4 \pi / 3,2 \pi / 3)$. Аналогично оценим вклад точки негладкости $(0,0)$ в асимптотику интеграла $J_{-}$. Функция

$$
B(\mathbf{p}):=3-\sqrt{3+2\left(\cos p_{1}+\cos p_{2}+\cos \left(p_{1}-p_{2}\right)\right)}
$$




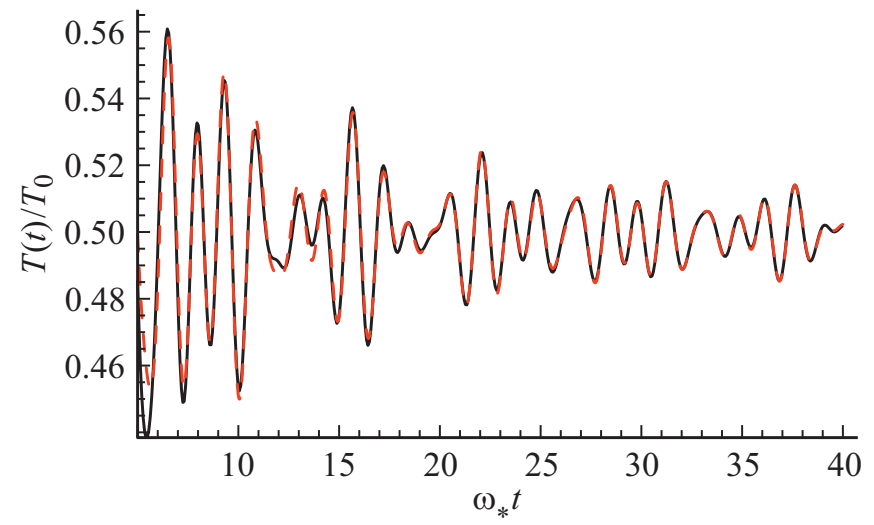

Зависимость $T(t) / T_{0}$ от $\omega_{*} t$.

гладкая вблизи начала координат, ее разложение Тейлора имеет вид

$$
B(\mathbf{p}):=\left(\left(p_{1}-p_{2} / 2\right)^{2}+3 p_{2}^{2} / 4\right) / 6+\ldots
$$

Заменяя переменные с помощью леммы Морса, получаем

$$
\int_{-\infty}^{+\infty} \int_{-\infty}^{+\infty} e^{2 i \omega_{ \pm} t} \chi(|\mathbf{p}|) \mathrm{d} p_{1} \mathrm{~d} p_{2}=\int_{0}^{+\infty} e^{2 i \omega_{*} r t} \mathfrak{F}(r) r \mathrm{~d} r
$$

Здесь $\mathfrak{F}$ - гладкая функция при $r>0$, которая аннулируется вдали от нуля. Применяя лемму Эрдейи, получаем, что последний интеграл убывает, как $O\left(t^{-2}\right)$. Таким образом, точки негладкости не дают вклада в главный член асимптотики $J_{ \pm}$и последний дается формулой $(16)$. Окончательно получаем

$$
\begin{gathered}
J_{+}(t)=\frac{6 \sqrt{2} \pi}{i \omega_{*} t} e^{2 i \sqrt{6} \omega_{*} t}+\frac{12 \pi}{\omega_{*} t} e^{4 i \omega_{*} t}+O\left(\frac{1}{\omega_{*}^{2} t^{2}}\right) ; \\
J_{-}(t)=\frac{6 \sqrt{2} \pi}{\omega_{*} t} e^{2 i \sqrt{2} \omega_{*} t}+O\left(\frac{1}{\omega_{*}^{2} t^{2}}\right)
\end{gathered}
$$

при $\omega_{*} t \rightarrow+\infty$. Поскольку

$$
T=\frac{T_{0}}{2}\left[1+\frac{1}{8 \pi^{2}} \operatorname{Re}\left(J_{+}(t)+J_{-}(t)\right)\right],
$$

отсюда следует, что

$$
\begin{aligned}
T(t)= & \frac{T_{0}}{2}\left[1+\frac{3 \sqrt{2}}{4 \pi \omega_{*} t}\left(\sin \left(2 \sqrt{6} \omega_{*} t\right)+\sqrt{2} \cos \left(4 \omega_{*} t\right)\right.\right. \\
& \left.\left.+\cos \left(2 \sqrt{2} \omega_{*} t\right)\right)\right]+O\left(\frac{1}{\omega_{*}^{2} t^{2}}\right), \quad \omega_{*} t \rightarrow+\infty .
\end{aligned}
$$

Зависимость $T(t) / T_{0}$ от $\omega_{*} t$ представлена на рисунке. Красным показан график асимптотического выражения (29), черным - результаты численного интегрирования для (26).

\section{8. Заключение}

Для широкого класса одномерных и двумерных скалярных гармонических решеток простой структуры получены асимптотические формулы, описывающие перераспределение кинетической и потенциальной энергий после мгновенного теплового возбуждения („быстрый процесс" ${ }^{\text {“ }}$. Асимптотики выводятся методом стационарной фазы из точных интегральных выражений, полученных в работах [24-26]. Асимптотики справедливы при больших по сравнению с характерным периодом колебаний атомов временах. По сравнению с интегральным представлениями, полученные выражения проще и позволяют аналитически описать характер затухания.

Согласно полученным результатам, разница кинетической и потенциальной энергий $L(t)$ испытывает затухающие степенным образом гармонические колебания. Показатель степенного затухания в типичном случае есть $d / 2$, где $d-$ размерность решетки. Частоты осцилляций определяются из дисперсионного соотношения $\omega(\mathbf{k})$ как удвоенные значения $\omega(\mathbf{k})$ в точках, в которых аннулируется групповая скорость $\mathrm{d} \omega / \mathrm{d} \mathbf{k}$. Для ряда одномерных (взаимодействия ближайших соседей, угловые взаимодействия, влияние упругого основания) и двумерных (квадратная и треугольная решетки с парным взаимодействием, квадратная решетка с угловым взаимодействием) скалярных решеток простой структуры асимптотические выражения выписаны явно. Показатели затухания и частоты осцилляций кинетической температуры, явно вычисленные в работе, сведены в таблицу.

Приведены примеры одномерных и двумерных решеток (см. примеры 4, 5 и 8), для которых затухание осцилляций $L(t)$ происходит медленнее, чем по закону $\sim t^{-d / 2}$. На примере одномерной цепочки показано, что необходимым условием медленного затухания является взаимодействие каждой из частиц решетки с большим числом соседей.

Предложенный алгоритм описания быстрых процессов допускает обобщение на скалярные и векторные решетки сложной структуры и различных размерностей. В качестве примера решетки сложной структуры рассмотрена гексагональная решетка (решетка графена), описаны колебания разницы кинетической и потенциальной энергий.

\section{Благодарности}

Автор выражает искреннюю благодарность А.М. Кривцову и В.А. Кузькину за постановку задачи и полезные обсуждения.

\section{Финансирование работы}

Работа выполнена при финансовой поддержке Российского научного фонда (грант № 18-11-00201). 


\section{Конфликт интересов}

Автор заявляет, что у него конфликта интересов.

\section{Список литературы}

[1] C. Boldrighini, A. Pellegrinotti, L. Triolo. J. Status. Phys. 30, 1,123 (1983).

[2] R.L. Dobrushin, A. Pellegrinotti, Yu.M. Suhov, L. Triolo. J. Status. Phys. 43, 3 (1986).

[3] T.V. Dudnikova, A.I. Komech, H. Spohn. J. Math. Phys. 44, 2596 (2003).

[4] T.V. Dudnikova, A.I. Komech. Russian J. Math. Phys. 12, 3, 301325 (2005)

[5] J.L. van Hemmen. Phys. Lett. 79A, 1 (1980).

[6] [BP.C. Hemmer. Dynamic and stochastic types of motion in the linear chain. Norges tekniske hoiskole (1959).

[7] B.L. Holian, W.G. Hoover, B. Moran, G.K. Straub. Phys. Rev. A 22, 2798 (1980).

[8] G. Klein, I. Prigogine. Physica 19, 1053 (1953).

[9] O.E. Lanford, J.L. Lebowitz. Time evolution and ergodic properties of harmonic systems. In: Lecture Notes in Physics. Springer, Berlin-Heidelberg-N.Y. (1975). V. 38. P. 144-177.

[10] S.L. Linn, H.S. Robertson. J. Phys. Chem. Solids 45, 2, 133 (1984).

[11] A. Mielke. Arch. Rat. Mech. Anal. 181, 401 (2006).

[12] H. Spohn, J.L. Lebowitz. Commun. Math. Phys. 54, 97 (1977).

[13] M.A. Huerta, H.S. Robertson, J.C. Nearing. J. Math. Phys. 12, 2305 (1971).

[14] V. Kannan, A. Dhar, J.L. Lebowitz. Phys. Rev. E 85, 041118 (2012).

[15] S. Lepri, C. Mejia-Monasterio, A. Politi. J. Phys. A 42, 2, 025001 (2008).

[16] Z. Rieder, J.L. Lebowitz, E. Lieb. J. Math. Phys. 8, 1073 (1967).

[17] А.М. Кривцов. Докл. АН 458, 3, 279 (2014).

[18] A.M. Krivtsov. On unsteady heat conduction in a harmonic crystal. arXiv:1509.02506 (2015).

[19] А.М. Кривцов. Докл. АН 464, 2, 162 (2015).

[20] М.Б. Бабенков, А.М. Кривцов, Д.В. Цветков. Физ. мезомеханика 19, 1, 60 (2016).

[21] В.А. Кузькин, А.М. Кривцов. Докл. АН 472, 5, 529 (2017).

[22] В.А. Кузькин, А.М. Кривцов. ФТТ 59, 5, 1023 (2017).

[23] V.A. Tsaplin, V.A. Kuzkin. Lett. Mater. 8, 1, 16 (2018).

[24] V.A. Kuzkin, A.M. Krivtsov. J. Phys.: Condens. Matter. 2, 505401 (2017).

[25] V.A. Kuzkin. Continuum Mech. Thermodyn. 31, 1401 (2019).

[26] V.A. Kuzkin. Continuum Mech. Thermodyn. 31, 1573 (2019).

[27] Г.И. Канель, С.В. Разоренов, А.В. Уткин, В.Е. Фортов. Ударноволновые явления в конденсированных средах. Янус-К, М. (1996). 407 с.

[28] B.L. Holian, W.G. Hoover, B. Moran, G.K. Straub. Phys. Rev. A 22, 2798 (198).

[29] B.L. Holian, M. Mareschal. Phys. Rev. E 82, 026707 (2010).

[30] W.G. Hoover, C.G. Hoover, K.P. Travis. Phys. Rev. Lett. 112, 144504 (2014).

[31] F. Silva, S.M. Teichmann, S.L. Cousin, M. Hemmer, J. Biegert. Nature Commun. 6, 6611 (2015).

[32] С.И. Ашитков, П.С. Комаров, М.Б. Агранат, Г.И. Канель, В.Е. Фортов. Письма в ЖЭТФ 98, 439 (2013).
[33] N.A. Inogamov, Yu.V. Petrov, V.V. Zhakhovsky, V.A. Khokhlov, B.J. Demaske, S.I. Ashitkov, K.V. Khishchenko, K.P. Migdal, M.B. Agranat, S.I. Anisimov, V.E. Fortov, I.I. Oleynik. AIP Conf. Proc. 1464, 593 (2012).

[34] K.V. Poletkin, G.G. Gurzadyan, J. Shang, V. Kulish. Appl. Phys. B 107, 137 (2012).

[35] D.A. Indeitsev, V.N. Naumov, B.N. Semenov, A.K. Belyaev. ZAMM 89, 2.

[36] М.В. Федорю. Метод перевала. Наука, М. (1977). 368 с.

Редактор Т.Н. Василевская 